\title{
Effect of Correlated Non-Gaussian Quadratures on the Performance of Binary Modulations
}

\author{
Valentine A. Aalo ${ }^{1}$ and George P. Efthymoglou ${ }^{2}$ \\ ${ }^{1}$ Department of Computer \& Electrical Engineering and Computer Science, Florida Atlantic University, Boca Raton, FL 33431, USA \\ ${ }^{2}$ Department of Digital Systems, University of Piraeus, 80 Karaoli and Dimitriou Street, 18534 Piraeus, Greece
}

Correspondence should be addressed to George P. Efthymoglou,gefthymo@unipi.gr

Received 14 January 2011; Accepted 31 March 2011

Academic Editor: Nikos Sagias

Copyright ( 2011 V. A. Aalo and G. P. Efthymoglou. This is an open access article distributed under the Creative Commons Attribution License, which permits unrestricted use, distribution, and reproduction in any medium, provided the original work is properly cited.

\begin{abstract}
The received signal in many wireless communication systems comprises of the sum of waves with random amplitudes and random phases. In general, the composite signal consists of correlated nonidentical Gaussian quadrature components due to the central limit theorem (CLT). However, in the presence of a small number of random waves, the CLT may not always hold and the quadrature components may not be Gaussian distributed. In this paper, we assume that the fading environment is such that the quadrature components follow a correlated bivariate Student-t joint distribution. Then, we derive the envelope distribution of the received signal and obtain new expressions for the exact and high signal-to-noise (SNR) approximate average BER for binary modulations. It also turns out that the derived envelope pdf approaches the Rayleigh and Hoyt distributions as limiting cases. Using the derived envelope pdf, we investigate the effect of correlated nonidentical quadratures on the error rate performance of digital communication systems.
\end{abstract}

\section{Introduction}

The wireless communication channel has attracted a lot of attention over the years as the need for higher data rates and spectral efficiency in mobile communications has increased. Recently, a unified probability density function (pdf) based on the generalized Laguerre polynomial that characterizes a wide range of distributions for small-scale fading has been derived in $[1,2]$ by modeling the received signal envelope as a nonlinear function of the modulus of the sum of an arbitrary number of multipath components. The unified envelope pdf covers many known Laguerre polynomial-series-based pdfs and multipath fading distributions, which include the multiple-waves-plus-diffuse-power (MWDP) fading [3], Nakagami- $m$ [4], Rician (Nakagami-n), Hoyt (Nakagami-q), Rayleigh, Weibull, $\kappa-\mu$ [5], and $\alpha-\mu$ (Stacy) [6] distributions as special cases. The existing analytical fading distributions have been derived considering both homogeneous and nonhomogeneous propagating environments. The MWDP fading model consists of $M$ specular constant-amplitude waves and a Gaussian-distributed diffused component. The $\kappa-\mu$ distribution considers a signal composed of clusters of multipath waves, with each cluster including a dominant component and scattered waves with identical powers. Moreover, the $\alpha-\mu$ fading model considers a signal composed of multipath waves propagating in a nonhomogeneous environment, where the resulting envelope is obtained as a nonlinear function of the modulus of the sum of the multipath components. Nakagami was the first to consider the case that the received signal is the sum of sinusoids with random amplitudes and derived the well-known Nakagami$m$ fading distribution, which includes Rayleigh fading as special case and it can also approximate well the Rician distribution.

Following the work by Nakagami, the sum of $M$ random vectors has attracted considerable attention $[7,8]$. Based on the central limit theorem (CLT), we know that this sum, for sufficiently large number of independent waves with uniform elementary phases, results in in-phase $(I)$ and quadrature (Q) components that follow Gaussian distributions with zero mean. The Rayleigh and Hoyt envelope pdfs can then be obtained assuming, respectively, identical and nonidentical variances (powers) for the quadrature components. However, the more general case of dependent (correlated) 
Gaussian quadratures with nonzero means and unequal variances was shown to provide good fit to measured propagation data in various wireless environments [7]. The case of correlated nonidentical $I$ and $Q$ Gaussian distributed components has not received much attention in the literature, as it does not yield an easy to handle analytical expression for the fading envelope pdf $[9,10]$.

When, as in an indoor fading environment, $M$ is not sufficiently large for the CLT to hold, the quadrature components may not be Gaussian distributed. Non-Gaussian quadratures have also been considered when $M$ is a random variable $[11,12]$. One distribution that has been used to model the quadrature components in such environment is the bivariate Student-t distribution [13]. In this paper we consider a general propagation environment that results in correlated, nonidentically distributed $I$ and $Q$ components in the received signal. Assuming correlation among the quadrature components and/or unequal variances, we derive the envelope pdf for the case of quadratures with correlated bivariate Student-t joint distribution. It is then shown that this envelope pdf closely approximates the envelope pdf of correlated Gaussian quadratures, derived in [9]. The two pdfs yield almost identical results for small values of envelope amplitudes. Based on this result, we are able to determine the parameters of this general fading model that affect the deep fade region and the corresponding bit error rate (BER) for binary digital modulation schemes. To the best of the authors' knowledge this analysis is novel.

The rest of the paper is organized as follows. In Section 2, we approximate the correlated Gaussian-distributed quadratures by the corresponding correlated Student-t-distributed quadratures and derive the resulting envelope pdf. In Section 3, analytical exact and high SNR approximate average BER expressions for binary modulations are derived for this general fading model. Numerical results that depict the effect of the statistical parameters on the BER performance are given in Section 4, whereas conclusions are drawn in Section 5.

\section{Envelope Distribution for Correlated Quadratures}

Let $\left(X_{c}, X_{s}\right)$ be a pair of correlated real Gaussian random variables with mean $\left(\mu_{c}, \mu_{s}\right)$ and variance $\left(\sigma_{c}^{2}, \sigma_{s}^{2}\right)$, respectively, and let $\rho=E\left[\left(X_{c}-\mu_{c}\right)\left(X_{s}-\mu_{s}\right)\right] / \sigma_{c} \sigma_{s}$ and $\lambda=\sigma_{c}^{2} / \sigma_{s}^{2}$ be the correlation coefficient and asymmetry power factor between $X_{c}$ and $X_{s}$, respectively. The complex Gaussian random variable $\left(X_{c}+j X_{s}\right)$ may be represented in polar form as $R e^{j \theta}$, where $R=\sqrt{X_{c}^{2}+X_{s}^{2}}$ and $\theta=\tan ^{-1}\left(X_{s} / X_{c}\right)$ are the envelope and phase, respectively. The joint pdf of the correlated Gaussian random variables $X_{c}$ and $X_{s}$ is given by [9]

$$
\begin{aligned}
p_{X_{c}, X_{s}}\left(x_{c}, x_{s}\right)= & \frac{1}{2 \pi \sigma_{c} \sigma_{s} \sqrt{1-\rho^{2}}} \\
& \times \exp \left\{-\frac{\left(\left(x_{c}-\mu_{c}\right) / \sigma_{c}\right)^{2}+\left(\left(x_{s}-\mu_{s}\right) / \sigma_{s}\right)^{2}-2 \rho\left(x_{c}-\mu_{c}\right)\left(x_{s}-\mu_{s}\right) / \sigma_{c} \sigma_{s}}{2\left(1-\rho^{2}\right)}\right\},
\end{aligned}
$$

and the corresponding envelope pdf has been derived in $[9$, equation (7) $]$. The envelope distribution in $[9$, equation (7)], which is based on the CLT argument, does not yield mathematically tractable receiver performances. Since in some practical fading environments the CLT argument may not always be valid (e.g., [13]), we replace (1) with the correlated bivariate Student-t distribution with $v$ degrees of freedom $(\mathrm{DOF})$, and the joint pdf of $\left(X_{c}, X_{s}\right)$ can then be written as $[11,14]$

$$
\begin{aligned}
& p_{X_{c}, X_{s}}\left(x_{c}, x_{s}\right)= \frac{1}{2 \pi \sigma_{c} \sigma_{s} \sqrt{1-\rho^{2}}} \\
& \times\left[1+\frac{\left(\left(x_{c}-\mu_{c}\right) / \sigma_{c}\right)^{2}+\left(\left(x_{s}-\mu_{s}\right) / \sigma_{s}\right)^{2}-2 \rho\left(x_{c}-\mu_{c}\right)\left(x_{s}-\mu_{s}\right) /\left(\sigma_{c} \sigma_{s}\right)}{2 \nu\left(1-\rho^{2}\right)}\right]^{-(v+1)}, \\
& v>0, x_{c}, x_{s} \geq 0 .
\end{aligned}
$$

Similar to the analysis in [9], in order to derive the pdf of the envelope $R$ when the quadrature components $X_{c}$ and $X_{s}$ have the joint pdf in (2), we consider the following linear transformation of the variables (which is equivalent to a rotation of the axis through the angle $\phi$ [15]):

$$
\begin{aligned}
& Y_{1}=X_{c} \cos \phi+X_{s} \sin \phi, \\
& Y_{2}=-X_{c} \sin \phi+X_{s} \cos \phi .
\end{aligned}
$$

It can be shown that if the angle of the rotation is chosen to be

$$
\phi=\frac{1}{2} \tan ^{-1}\left(\frac{2 \rho \sigma_{c} \sigma_{s}}{\sigma_{c}^{2}-\sigma_{s}^{2}}\right),
$$

then the random variables $Y_{1}$ and $Y_{2}$ are uncorrelated [15]. The mean values of $Y_{1}$ and $Y_{2}$ are given by

$$
\begin{aligned}
& \mu_{1}=E\left[Y_{1}\right]=\mu_{c} \cos \phi+\mu_{s} \sin \phi, \\
& \mu_{2}=E\left[Y_{2}\right]=\mu_{s} \cos \phi-\mu_{c} \sin \phi .
\end{aligned}
$$


Similarly, their variances are

$$
\begin{aligned}
& \sigma_{1}^{2}=\operatorname{var}\left[Y_{1}\right]=\sigma_{c}^{2} \cos ^{2} \phi+\sigma_{s}^{2} \sin ^{2} \phi+\rho \sigma_{c} \sigma_{s} \sin 2 \phi, \\
& \sigma_{2}^{2}=\operatorname{var}\left[Y_{2}\right]=\sigma_{c}^{2} \sin ^{2} \phi+\sigma_{s}^{2} \cos ^{2} \phi-\rho \sigma_{c} \sigma_{s} \sin 2 \phi .
\end{aligned}
$$

Note that since $Y_{1}$ and $Y_{2}$ are uncorrelated, it follows that $\left(Y_{1}-\mu_{1}\right)$ and $\left(Y_{2}-\mu_{2}\right)$ are orthogonal. Therefore by expressing (2) in polar form, the envelope pdf is given by

$p_{R}(r)$

$$
\begin{aligned}
& =\frac{(2 \nu)^{v+1} r}{2 \pi \sigma_{1} \sigma_{2}} \int_{0}^{2 \pi}\left[2 \nu+\left(\frac{r \cos \psi-\mu_{1}}{\sigma_{1}}\right)^{2}+\left(\frac{r \sin \psi-\mu_{2}}{\sigma_{2}}\right)^{2}\right]^{-(\nu+1)} d \psi \\
& =\frac{(2 \nu)^{v+1} r}{2 \pi \sigma_{1} \sigma_{2}} \int_{0}^{2 \pi} \frac{d \psi}{\left[c+d \cos ^{2} \psi-(e \cos \psi+f \sin \psi)\right]^{(\nu+1)}},
\end{aligned}
$$

where $\psi=\theta-\phi, c=2 \nu+\mu_{1}^{2} / \sigma_{1}^{2}+\mu_{2}^{2} / \sigma_{2}^{2}+r^{2} / \sigma_{2}^{2}, d=$ $r^{2}\left(1 / \sigma_{1}^{2}-1 / \sigma_{2}^{2}\right), e=2 \mu_{1} r / \sigma_{1}^{2}, f=2 \mu_{2} r / \sigma_{2}^{2}$. In order to make our analysis tractable, we restrict it to the special case of zero means for the quadrature components, that is, $\mu_{c}=\mu_{s}=0$ (implying $\mu_{1}=\mu_{2}=0$ ). It then follows that $c=2 v+r^{2} / \sigma_{2}^{2}$, $e=f=0$, and (7) reduces to

$$
p_{R}(r)=\frac{(2 \nu)^{v+1} r}{2 \pi \sigma_{1} \sigma_{2}} \int_{0}^{2 \pi} \frac{d \psi}{\left[c+d \cos ^{2} \psi\right]^{(\nu+1)}}
$$

The integral in (8) can be evaluated using [16, equation (3.682)], and after some mathematical manipulations, the result for the envelope pdf is given as

$p_{R}(r)=\frac{r}{\sigma_{1} \sigma_{2}\left(1+r^{2} /\left(2 \nu \sigma_{2}^{2}\right)\right)^{\nu+1}}{ }_{2} F_{1}\left(\frac{1}{2}, \nu+1 ; 1 ; \frac{\left(1-\sigma_{2}^{2} / \sigma_{1}^{2}\right)}{1+2 \nu \sigma_{2}^{2} / r^{2}}\right)$,

where ${ }_{2} F_{1}(a, b ; c ; x)$ is the Gaussian hypergeometric function defined in [17, equation (15.1.1)].

\subsection{Special Cases}

(1) When $v \rightarrow \infty$, using the relations in [17, equation (4.2.21)] and [17, equation (13.6.3]), (9) gives

$$
\begin{aligned}
p_{R}^{(v \rightarrow \infty)}(r)= & \lim _{\nu \rightarrow \infty}\left[\left(\frac{r}{\sigma_{1} \sigma_{2}\left(1+r^{2} /\left(2 \nu \sigma_{2}^{2}\right)\right)^{\nu+1}}\right)\right] \\
& \times{ }_{2} F_{1}\left(\frac{1}{2}, \nu+1 ; 1 ; \frac{r^{2}}{2 \nu}\left(\frac{1}{\sigma_{2}^{2}}-\frac{1}{\sigma_{1}^{2}}\right)\right) \\
= & \frac{r}{\sigma_{1} \sigma_{2}} \exp \left(-\frac{r^{2}}{2 \sigma_{2}^{2}}\right) \exp \left(\frac{r^{2}}{4}\left(\frac{1}{\sigma_{2}^{2}}-\frac{1}{\sigma_{1}^{2}}\right)\right) \\
& \times I_{0}\left(\frac{r^{2}}{4}\left(\frac{1}{\sigma_{2}^{2}}-\frac{1}{\sigma_{1}^{2}}\right)\right) \\
= & \frac{r}{\sigma_{1} \sigma_{2}} \exp \left(-\frac{r^{2}}{4}\left(\frac{1}{\sigma_{2}^{2}}-\frac{1}{\sigma_{1}^{2}}\right)\right) I_{0}\left(\frac{r^{2}}{4}\left(\frac{1}{\sigma_{2}^{2}}-\frac{1}{\sigma_{1}^{2}}\right)\right),
\end{aligned}
$$

which corresponds to the Hoyt envelope distribution [9], as expected.

(2) For equal variances of the quadrature components (i.e., $\left.\sigma_{c}^{2}=\sigma_{s}^{2}=\sigma^{2}\right)$, we have $\sigma_{1}^{2}=\sigma^{2}(1+\rho)$ and $\sigma_{2}^{2}=\sigma^{2}(1-\rho)$, and $(9)$ can also be expressed as

$$
\begin{aligned}
p_{R}^{(\lambda=1)}(r)= & \frac{r}{\sigma^{2} \sqrt{1-\rho^{2}}\left(1+r^{2} / \mu\right)^{\nu+1}} \\
& \times{ }_{2} F_{1}\left(\frac{1}{2}, \nu+1 ; 1 ; \frac{\eta}{1+\mu / r^{2}}\right),
\end{aligned}
$$

where $\mu=2 \sigma^{2} \nu(1-\rho)$ and $\eta=2 \rho /(1+\rho)$.

(3) When the quadrature components are also uncorrelated, that is, $\rho=0$, (11) further reduces to

$$
p_{R}^{(\lambda=1, \rho=0)}(r)=\frac{r}{\sigma^{2}} \frac{1}{\left(1+r^{2} /\left(2 \sigma^{2} \nu\right)\right)^{\nu+1}} .
$$

Moreover, using the fact that $\lim _{v \rightarrow \infty}\left\{1 /(1+x /(k v))^{\nu+1}\right\}=$ $e^{-x / k}[16]$, it follows that when $v \rightarrow \infty$ (12) approaches the Rayleigh distribution with average power $2 \sigma^{2}$, as expected.

\section{BER Analysis}

For binary modulation schemes, the BER in AWGN conditioned on the instantaneous SNR $\gamma$ can be written in a compact form as [18, equation (8.100)]

$$
P_{E}(\gamma)=\frac{\Gamma(b, a \gamma)}{2 \Gamma(b)},
$$

where parameters $a$ and $b$ depend on the type of modulation/detection scheme and take the values $(a=1, b=$ $1 / 2$ ) for coherent BPSK, $(a=1 / 2, b=1 / 2)$ for coherent binary frequency shift keying (BFSK), $(a=1, b=1)$ for differentially coherent BPSK, and $(a=1 / 2, b=1)$ for noncoherent BFSK. In $(13), \Gamma(\cdot, \cdot)$ denotes the upper incomplete gamma function [16, equation (8.350.2)]. Due to the presence of fading, the instantaneous received SNR is given by $\gamma=\left(E_{b} / N_{0}\right) R^{2}$, where $R$ is the instantaneous fading amplitude, $E_{b}$ is the energy per bit, and $N_{0}$ is the one-sided noise power spectral density. Using the envelope pdf in (9), the pdf of the instantaneous SNR is given by

$$
p_{\gamma}(\gamma)=\frac{\nu}{\kappa \beta(1+\gamma / \beta)^{\nu+1}}{ }_{2} F_{1}\left(\frac{1}{2}, \nu+1 ; 1 ; \frac{1-\left(1 / \kappa^{2}\right)}{1+\beta / \gamma}\right),
$$

where the average received SNR per bit is $\bar{\gamma}=\left(E_{b} / N_{0}\right) E\left[R^{2}\right]$, with $E\left[R^{2}\right]=\sigma_{c}^{2}+\sigma_{s}^{2}=\sigma_{1}^{2}+\sigma_{2}^{2}$ being the average channel power. In (14), $\kappa \triangleq \sqrt{\sigma_{1}^{2} / \sigma_{2}^{2}}$ is defined as the square root of the ratio of the $I$ and $Q$ signal powers after the rotation with the angle $\phi$ and $\beta \triangleq 2 v \bar{\gamma} /\left(1+\kappa^{2}\right)$. Note that the effect of both correlation and unequal variances in the quadratures is included in the value of the asymmetry factor $\kappa$. In particular, for uncorrelated quadratures $(\rho=0)$ with equal powers $(\lambda=$ $1)$, we have $\kappa=1$. 
In a flat-fading environment, the average BER for binary modulations is given by averaging the BER in (13) over the pdf of $\gamma$, as

$$
\bar{P}_{E}=\int_{0}^{\infty} P_{E}(\gamma) p_{\gamma}(\gamma) d \gamma .
$$

Making the change of variables $x=a \gamma, \bar{P}_{E}$ is given by

$$
\begin{aligned}
\bar{P}_{E}= & \frac{v}{2 \kappa a \beta \Gamma(b)} \int_{0}^{\infty} \Gamma(b, x) \frac{1}{(1+x /(a \beta))^{\nu+1}} \\
& \times{ }_{2} F_{1}\left(\frac{1}{2}, \nu+1 ; 1 ; \frac{1-\left(1 / \kappa^{2}\right)}{1+a \beta / x}\right) d x .
\end{aligned}
$$

Expressing the complementary incomplete gamma function in terms of Meijer's $G$-function [16]

$$
\Gamma(b, x)=G_{1,2}^{2,0}\left(\begin{array}{l|c}
x & 1 \\
b, 0
\end{array}\right)
$$

and using the series expansion for the hypergeometric function, (16) becomes

$$
\begin{aligned}
\bar{P}_{E}= & \frac{\nu(a \beta)^{\nu}}{2 \kappa \Gamma(b)} \sum_{n=0}^{\infty} \frac{(1 / 2)_{n}(\nu+1)_{n}\left[1-\left(1 / \kappa^{2}\right)\right]^{n}}{(1)_{n} n !} \\
& \times \int_{0}^{\infty} x^{n}(x+a \beta)^{-(n+\nu+1)} G_{1,2}^{2,0}\left(\begin{array}{c|c}
x & 1 \\
b, 0
\end{array}\right) d x,
\end{aligned}
$$

where $(v)_{n}=\Gamma(v+n) / \Gamma(v)$ denotes the Pochhammer symbol [16]. The integral in (18) may be solved using [16, equation $(7.811 .5)$ ], to obtain the average BER for binary modulations, as

$$
\begin{aligned}
& \bar{P}_{E}=\frac{1}{2 \kappa \Gamma(b) \Gamma(v)}
\end{aligned}
$$

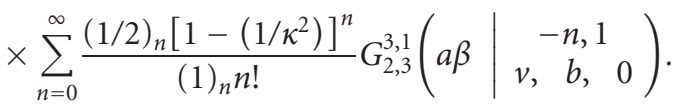

Although the BER expression (19) is given as an infinite series form, it converges rapidly and steadily, requiring few terms for an accurate result. Therefore (19) can be easily evaluated using standard mathematical programs such as Mathematica. Since the summation (19) converges very fast, no more than five terms were used for good numerical accuracy.

\subsection{Special Cases}

(1) For the case of noncoherent/differentially coherent detection $(b=1),(19)$ reduces to

$$
\begin{aligned}
\bar{P}_{E, b=1}= & \frac{1}{2 \kappa \Gamma(v)} \\
& \times \sum_{n=0}^{\infty} \frac{(1 / 2)_{n}\left[1-\left(1 / \kappa^{2}\right)\right]^{n}}{(1)_{n} n !} G_{1,2}^{2,1}\left(a \beta \mid \begin{array}{c}
-n \\
v, 0
\end{array}\right),
\end{aligned}
$$

where where we used the property [19, equation (07.34.03.0002.01)], that is,

$$
G_{2,3}^{3,1}\left(\begin{array}{l|c|c}
a \beta & -n, 1 \\
v, & 1, & 0
\end{array}\right)=G_{1,2}^{2,1}\left(\begin{array}{l|c}
a \beta & -n \\
v, & 0
\end{array}\right) .
$$

(2) For the uncorrelated quadratures with equal variances, that is, $\kappa=1,(19)$ reduces to

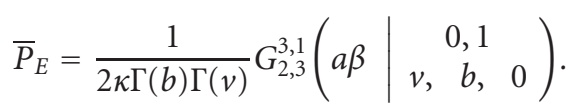

(3) By defining $x=\gamma / \bar{\gamma}$, we can approximate $p_{x}(x) \rightarrow$ $\zeta x^{t}+o\left(x^{t}\right)$, for $x \rightarrow 0^{+}$. For the SNR pdf given in (14), by taking the $\lim _{x \rightarrow 0} p_{x}(x)$ we can easily show that

$$
\begin{aligned}
& \zeta=\frac{\left(1+\kappa^{2}\right)}{2 \kappa}, \\
& t=0 .
\end{aligned}
$$

Therefore, the average BER of binary modulations for high SNR values is given by [20]

$$
\begin{aligned}
\bar{P}_{E}^{\infty} & =\frac{1}{2 \Gamma(b)} \int_{0}^{\infty} \Gamma(b, a \bar{\gamma} x)[\zeta+o(x)] d x \\
& =\frac{\zeta \Gamma(b+1)}{2 \Gamma(b)}(a \bar{\gamma})^{-1}+o\left(\bar{\gamma}^{-1}\right) \\
& =\frac{\left(1+\kappa^{2}\right) b}{4 \kappa}(a \bar{\gamma})^{-1}+o\left(\bar{\gamma}^{-1}\right) .
\end{aligned}
$$

Equation (24) implies that the diversity order is $G_{d}=$ 1 and the coding gain is given by $G_{c}=4 \kappa a /\left(\left(1+\kappa^{2}\right) b\right)$. We observe that the expression of the asymptotic BER for this fading model is controlled only by factor $\kappa$, which includes the effect of correlation and power ratio of the quadratures. Note that for uncorrelated quadratures with equal variances $(\kappa=1)$ the coding gain is $G_{c}=4 a /(2 b)$, as expected [20].

\section{Numerical Results}

In this section we present numerical results to show the effect of statistical parameters $(v, \rho, \lambda)$ on the envelope pdf of the received signal and on the corresponding BER of binary digital modulations. Although not shown here due to space limitations, we have observed that small values of $v$ change somehow the shape of the envelope pdf compared to high values (i.e., $v \geq 10$ ), but they do not affect the deep fade region. This result was indicated by the absence of parameter $v$ in the expression of the asymptotic result for the average BER. For this reason we assume $v=5$ in the plots.

Figure 1 plots the envelope pdf given in (9) for $\sigma_{c}=$ 1 and for different values of the correlation coefficient $\rho$ and asymmetry power factor $\lambda$ (yielding different values of asymmetry power factor $\kappa$ ). The figure shows that nonzero correlation coefficients $\rho$ and high values of $\lambda$ (i.e., conditions that yield high values of $\kappa$ ) result in higher probability values 


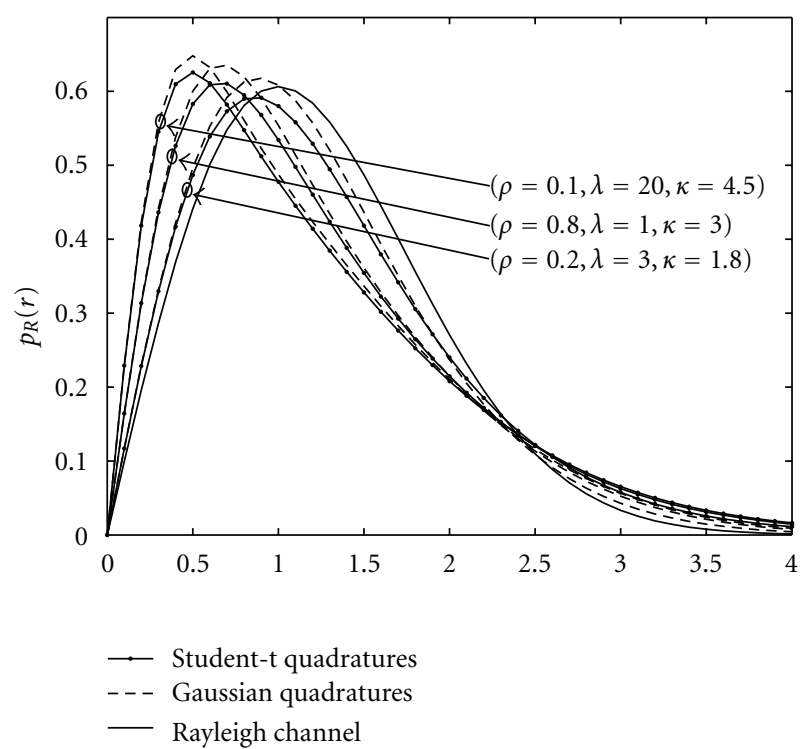

Figure 1: Envelope pdf of correlated bivariate Student-t and Gaussian quadratures for $\sigma_{c}=1$ and for several values of $(\rho, \lambda, \kappa)$.

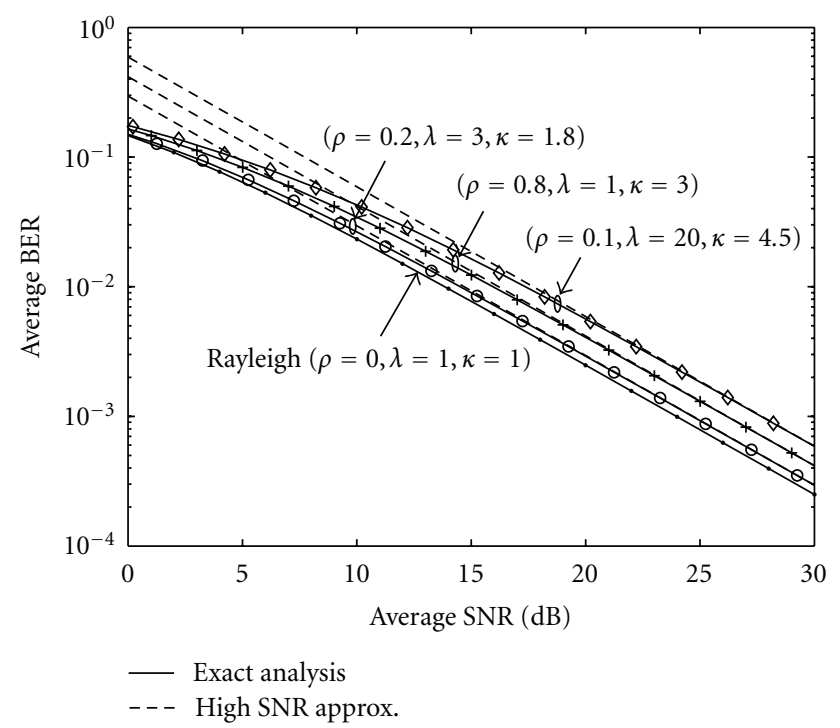

FIgUre 2: Exact and asymptotic BER for coherent BPSK with correlated bivariate Student-t quadratures for $\sigma_{c}=1$ and for several values of $(\rho, \lambda, \kappa)$.

over the deep fade region, as indicated by the shift to the left of the corresponding pdf curve. The modeling of the deep fade region is important since it is known to severely affect the performance of wireless communication systems.

To illustrate the effect of this fading model, Figure 2 plots the average BER of coherent BPSK for different statistical parameters $(\rho, \lambda, \kappa)$ and compares the exact and high SNR approximate results. Note that the summation in (19) converges very fast and no more than five terms were needed to give accurate numerical results. Moreover, we can see that the asymptotic BER result in (24) successfully predicts the high SNR slope (diversity order), which is equal to 1 , and the coding gain, yielding an excellent agreement with the exact average BER at high SNRs. From this figure, we observe that the error performance is affected considerably by the statistical parameter values. This is verified by the asymptotic analysis which shows the influence of $\kappa$ on the coding gain of the asymptotic BER. Therefore, using the proposed analysis for this fading model, we can quantify the effect of these statistical parameters on the error rate performance of binary digital communication systems.

\section{Conclusion}

In this letter, by approximating the correlated bivariate Gaussian joint pdf by the corresponding Student-t joint distribution, we derived the envelope pdf for the case of quadrature components with zero means and nonidentical variances. We showed that the deep fade region is controlled by the amount of correlation between the quadratures as well as their power ratio, and we derived expressions for the exact and asymptotic BER performance for binary digital modulations operating in this statistical fading channel model.

\section{References}

[1] C. C. Chai and T. T. Tjhung, "Unified Laguerre polynomialseries-based distribution of small-scale fading envelopes," IEEE Transactions on Vehicular Technology, vol. 58, no. 8, pp. 3988-3999, 2009.

[2] Y. Sun, A. Baricz, and S. Zhou, "Corrections to "Unified Laguerre polynomial-series-based distribution of small-scale fading envelopes"', IEEE Transactions on Vehicular Technology, vol. 60 , no. 1, pp. 347-349, 2011.

[3] G. D. Durgin, T. S. Rappaport, and D. A. de Wolf, "New analytical models and probability density functions for fading in wireless communications," IEEE Transactions on Communications, vol. 50, no. 6, pp. 1005-1015, 2002.

[4] M. Nakagami, "The $m$-distribution, a general formula of intensity distribution of rapid fading," in Statistical Methods in Radio Wave Propagation, W. G. Hoffman, Ed., Pergamon Press, Oxford, UK, 1960.

[5] M. D. Yacoub, "The $\kappa-\mu$ distribution and the $\eta-\mu$ distribution," IEEE Antennas and Propagation Magazine, vol. 49, no. 1, pp. 68-81, 2007.

[6] M. D. Yacoub, "The $\alpha-\mu$ distribution: a physical fading model for the Stacy distribution," IEEE Transactions on Vehicular Technology, vol. 56, no. 1, pp. 27-34, 2007.

[7] A. Abdi, H. Hashemi, and S. Nader-Esfahani, "On the PDF of the sum of random vectors," IEEE Transactions on Communications, vol. 48, no. 1, pp. 7-12, 2000.

[8] A. Abdi, "Comments on "a new theoretical model for the prediction of rapid fading variations in an indoor environment'," IEEE Transactions on Vehicular Technology, vol. 50, no. 1, pp. 331-334, 2001.

[9] V. A. Aalo, G. P. Efthymoglou, and C. Chirasil, "On the envelope and phase distributions for correlated Gaussian quadratures," IEEE Communications Letters, vol. 11, no. 12, pp. 985-987, 2007.

[10] P. Beckmann and A. Spizzichino, The Scattering of Electromagnetic Waves from Rough Surfaces, Pergamon Press, Oxford, UK, 1963. 
[11] P. Beckmann, Probability in Communication Engineering, Brace and World, San Diego, Calif, USA, 1967.

[12] E. Jakeman, "On the statistics of K-distributed noise," Journal of Physics A, vol. 13, no. 1, pp. 31-48, 1980.

[13] D. S. Polydorou and C. N. Capsalis, "A new theoretical model for the prediction of rapid fading variations in an indoor environment," IEEE Transactions on Vehicular Technology, vol. 46, no. 3, pp. 748-754, 1997.

[14] K. Fang, S. Kotz, and K. Ng, Symmetric Multivariate and Related Distributions, Chapman and Hall, London, UK, 1990.

[15] S. J. Press, Applied Multivariate Analysis, Holt, Rinehart and Winston, New York, NY, USA, 1972.

[16] I. S. Gradshteyn and I. M. Ryzhik, Table of Integrals, Series and Products, Academic Press, New York, NY, USA, 5 edition, 1980.

[17] M. Abramowitz and I. Stegun, Handbook of Mathematical Functions, Dover, New York, NY, USA, 1970.

[18] M. K. Simon and M. S Alouini, Digital Communication over Fading Channels, Wiley, New York, NY, USA, 2 edition, 2005.

[19] “The Wolfram Functions Site," http://functions.wolfram.com/.

[20] Z. Wang and G. B. Giannakis, "A simple and general parameterization quantifying performance in fading channels," IEEE Transactions on Communications, vol. 51, no. 8, pp. 13891398, 2003. 

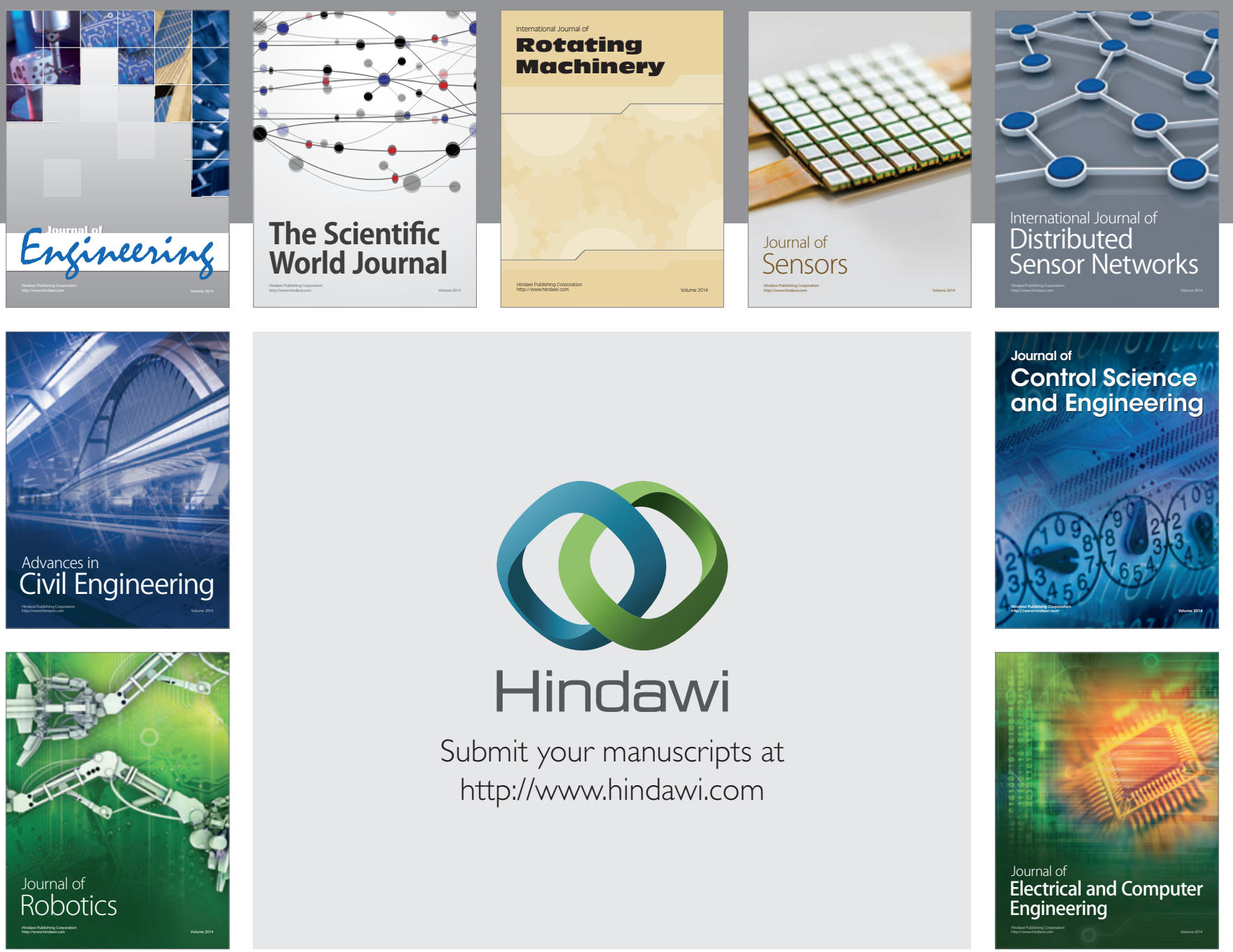

Submit your manuscripts at

http://www.hindawi.com
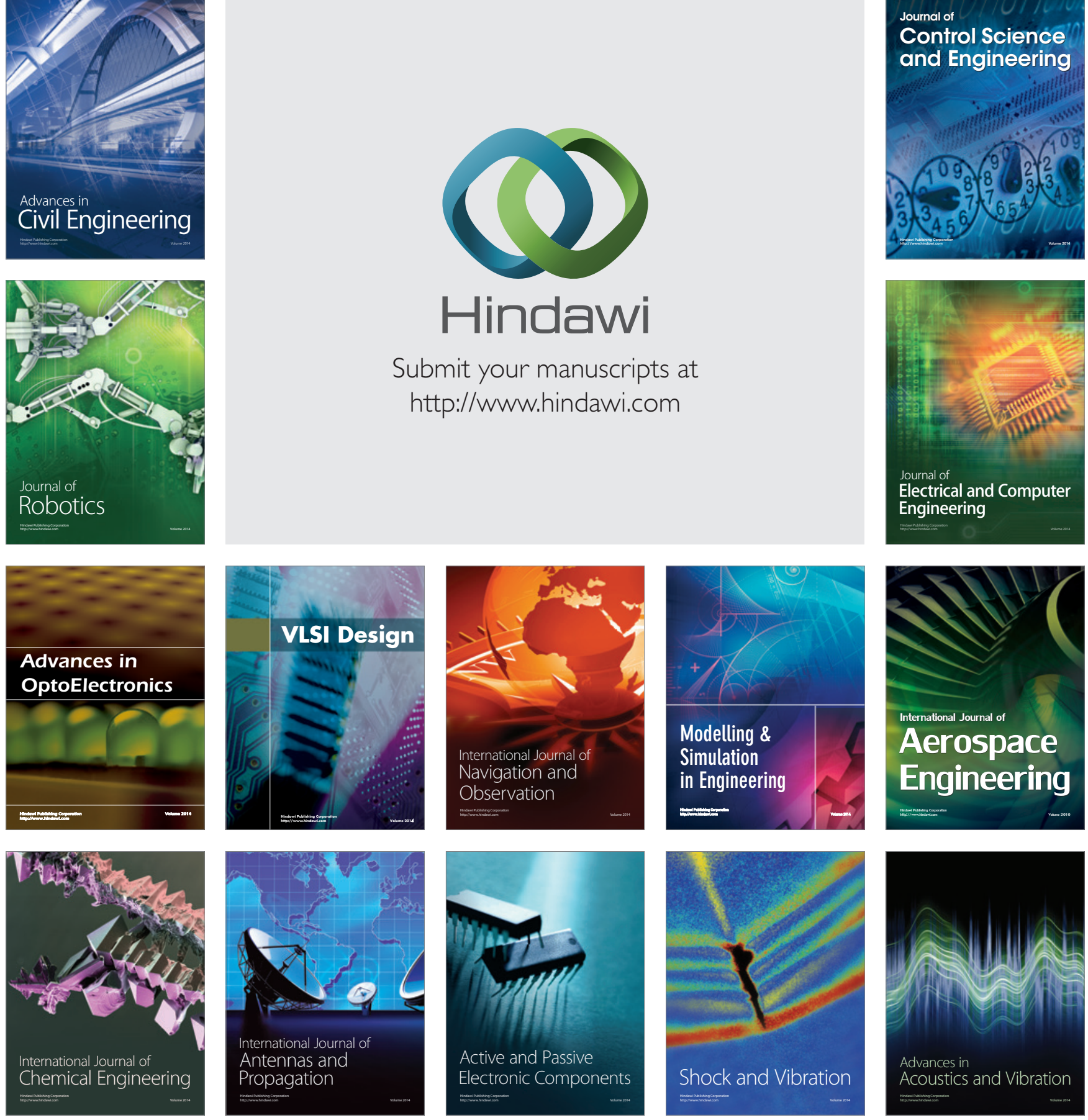